East African Medical Journal Vol. 83 No. 10 October 2006

NEOVASCULAR GLAUCOMA IN A NIGERIAN AFRICAN POPULATION:

A.O. Ashaye, FWACS, MSc, Department of Ophthalmology, College of Medicine, University of Ibadan, Oyo State, Nigeria and C.O. Adeoti, FWACS, FMCOPhth, Department of Ophthalmology, College of Health Sciences, Ladoke Akintola College of Technology, Osogbo, Nigeria

Request for reprints to: Dr. A.O. Ashaye, Department of Ophthalmology, College of Medicine, University of Ibadan, Oyo State, Nigeria

\title{
NEOVASCULAR GLAUCOMA IN A NIGERIAN AFRICAN POPULATION
}

\author{
A.O. ASHAYE and C.O. ADEOTI
}

\begin{abstract}
Objective: To determine the ocular and systemic factors associated with neovascular glaucoma (NVG) in an African population.

Design: Hospital based cross sectional study.

Setting: Eye clinic, Department of Ophthalmology, University College Hospital, Ibadan, Nigeria. Subjects: Sixty one consecutive patients with clinical diagnosis of NVG seen between January 1995 and December 1999 had a complete ocular evaluation.

Results: Among the 61 subjects studied with an identifiable aetiological factor presumably causing neovascular glaucoma, $82 \%$ had associated posterior segment diseases producing ischaemia. These were retinal venous obstruction $(78.7 \%)$, retinal arterial occlusion $(1.6 \%)$. Those with no identifiable vaso-occlusive disease had couching (11.5\%), aphakia with vitreous loss $(3.3 \%)$ and chronic uveitis (1.6\%). Systemic arterial hypertension was present in $62.3 \%$ while diabetes mellitus was present in only $8.3 \%$ of the subjects studied. Chronic uveitis and penetrating eye injury were infrequently diagnosed. Chronic open angle glaucoma was present in the other eye of $37.7 \%$ of subjects.

Neovascular glaucoma was unilateral in $95.1 \%$ of subjects. $84.4 \%$ of affected eyes were blind on presentation. Males outnumbered females among subjects with NVG above 40 years, while females outnumbered males in the subjects below 40 years of age. Eyes of that were couched constitute a significant proportion of subjects with neovascular glaucoma.

Conclusion: Medical conditions such as systemic hypertension, diabetes and ocular conditions like retinal vein, retinal artery occlusion, couching and glaucoma were associated with NVG. Most of these ocular and systemic associations should be identified early and treated to prevent neovascular glaucoma in the other eye of the subject.
\end{abstract}

\section{INTRODUCTION}

Blindness from primary and secondary glaucoma have been little addressed in most developing countries. The slowly progressive blindness from primary open angle glaucoma has received slightly more attention than the secondary glaucomas. Neovascular glaucoma (NVG) is such type of secondary glaucoma, and it results from many disease processes that cause ischaemia to the eye.
The fact that neovascular glaucoma frequently follows retinal vein occlusion is well known (1-8). Other aetiological associations with NVG include diabetic retinopathy, central retinal artery occlusions, ocular inflammation, injury and intraocular tumours $(3,6,7)$.

Neovascular glaucoma carries a guarded prognosis. Prognosis is dependent on the degree of the resulting ischaemia and early treatment of NVG. Treatment of the underlying ocular and systemic 
diseases may reduce the occurrence in the other eye (8-11).

In developing countries especially, where there are delays in diagnosis or non-availability of appropriate equipments for the management, NVG is a potentially devastating disease. It cannot only lead to loss of vision but of the globe itself.

It has been recognised that racial and geographic variations occur in the incidence and mortality from ischaemic conditions affecting the brain such as cerebrovascular diseases (CVD) which is providing a key to understanding the risk factors for CVD (9). There is paucity of information on NVG which is an ocular ischaemic condition in Africans, this paucity of information however does not allow comparison of risk factors for NVG between Africans and other races.

This study aims to determine the demographic characteristics, aetiological associations of NVG in African subjects presenting with neovascular glaucoma in a tertiary hospital in South Western Nigeria.

\section{MATERIALS AND METHODS}

A study of patients who primarily presented with neovascular glaucoma (NVG) to the Eye Clinic of the University College Hospital (UCH), Ibadan, Nigeria between January 1995 and December 1999 was carried out. Diagnosis of NVG was made based on clinical assessments which were the observation of any of the following features, optic nerve damage associated with (a) the presence of vessels or capillaries on the iris, pupil margin under slit lamp magnification or at the anterior chamber angle with gonioscopy with (b) elevated intraocular pressure (lOP) higher than $21 \mathrm{mmHg}$. (c) This could be accompanied by acute, severe pain, headache, photophobia, reduced vision, conjunctival injection and corneal oedema with or without hyphaema.

Each patient had a complete ophthalmological assessment including visual acuity, slit lamp examination, gonioscopy, ophthalmoscopy when possible and IOP measurements.

The demographic characteristics of the patients were noted. All patients had blood pressure measurements and urinalysis for sugar and protein. Hypertension was defined as systolic blood pressure (SBP) greater than $140 \mathrm{mmHg}$ and/or diastolic blood pressure (DBP) $90 \mathrm{mmHg}$ and over, or if patient was already receiving antihypertensive medications. Diabetes Mellitus was diagnosed if the patient had glycosuria or was on medication for diabetes. Chronic glaucoma was diagnosed in the other eye when there was optic disc cupping greater than $0.5 \mathrm{mmHg}$, visual field defects with or without intraocular pressure greater than $21 \mathrm{mmHg}$. Diagnosis of vein occlusions, arterial occlusions were made from fundoscopic features. Statistical analyses were performed using the chi-square test.

\section{RESULTS}

Sixty six patients were diagnosed to have NVG out of a total of 16,500 constituting $0.4 \%$ of all new out patient attendance to the eye clinic during the period under review. This represented $1.0 \%$ of all glaucomas seen. Five subjects with incomplete information were excluded from data analysis. Of the remaining sixty one subjects, thirty two subjects $(52.5 \%)$ were males. Mean age at presentation was 59.2 years [SD 12.7 years]. Age range was 31-84 years, four patients were under 40 years of age. Bilateral NVG occurred in three subjects $(4.9 \%)$ while unilateral NVG occurred in 58 (95.1\%) of the subjects. The right eye and left eyes were affected almost equally, right eye was affected in $50.8 \%$ of cases. Among the subjects aged 40-79 years, males with NVG outnumbered females while among the subjects $30-39$ years and those aged 80 years and above, females outnumbered males (Table 1).

The main presumed aetiological factor associated with NVG in the subjects studied was retinal vein occlusion occurring in $48(78.7 \%)$ of the subjects. Of these, central retinal vein occlusion accounted for $59.0 \%$, hemicentral retinal vein occlusion (HCRVO) $16.4 \%$, branch retinal vein occlusion (BRVO) 3.3\%, central retinal artery occlusion (CRAO) $1.6 \%$, diabetic retinopathy was $1.6 \%$. Others were ocular inflammation which were associated with couching $(11.5 \%)$, uveitis with retinal detachment $(1.6 \%)$ aphakia with vitreous loss (3.3\%) and penetrating eye injury $(1.6 \%)$ (Table 2). Of the three subjects who had bilateral NVG, one subject had DR while the other two had systemic hypertension. 
Table 1

Demographic and clinical characteristics of subjects with NVG

\begin{tabular}{|c|c|c|c|c|c|c|c|c|c|c|c|c|c|}
\hline & \multicolumn{2}{|c|}{ All } & \multicolumn{2}{|c|}{ CRVO } & \multicolumn{2}{|c|}{ HCRVO } & \multicolumn{2}{|c|}{ BRVO } & \multicolumn{2}{|c|}{ CRAO } & \multicolumn{2}{|c|}{$\begin{array}{c}\text { Diabetic } \\
\text { Retinopathy }\end{array}$} & Others \\
\hline & No. & $(\%)$ & No. & $(\%)$ & No. & $(\%)$ & No. & $(\%)$ & No. & $(\%)$ & No. & $(\%)$ & No. $(\%)$ \\
\hline \multicolumn{14}{|l|}{ Characteristics } \\
\hline $\begin{array}{l}\text { Total } \\
\text { Age (years) }\end{array}$ & 61 & 100 & 36 & 59.0 & 10 & 16.4 & 2 & 3.3 & 1 & 1.6 & 1 & 1.6 & $11 \quad 18.0$ \\
\hline$<40$ & 4 & 6.6 & 3 & 8.3 & - & - & & - & - & - & & - & 1 \\
\hline $40-49$ & 11 & 18.0 & 5 & 13.9 & 2 & 20 & & - & - & - & & - & 4 \\
\hline $50-59$ & 11 & 18.0 & 5 & 13.9 & 4 & 40.0 & & 1 & - & - & & - & 1 \\
\hline $60-69$ & 20 & 32.8 & 14 & 38.9 & 1 & 10.0 & & 1 & 1 & 1 & & 1 & 2 \\
\hline $70-79$ & 11 & 18.0 & 7 & 19.7 & 3 & 30.0 & & 1 & - & - & & - & 1 \\
\hline$>80$ & 4 & 6.6 & 2 & 5.6 & & - & & - & - & - & & - & 2 \\
\hline Total & 61 & 100 & 36 & - & & 10 & & 2 & 1 & 1 & & 1 & - \\
\hline $\begin{array}{l}\text { Mean age } \\
\text { (years) }\end{array}$ & \multicolumn{2}{|c|}{59.2} & \multicolumn{2}{|c|}{59.2} & \multicolumn{2}{|c|}{59.5} & \multicolumn{2}{|c|}{61.0} & \multicolumn{2}{|c|}{-} & & - & 55.3 \\
\hline \multicolumn{14}{|l|}{ Sex } \\
\hline Male & 32 & 52.5 & \multicolumn{2}{|c|}{18} & & 6 & & 1 & \multicolumn{2}{|c|}{1} & & - & 6 \\
\hline Female & 29 & 47.5 & \multicolumn{2}{|c|}{18} & & 4 & & 1 & - & - & & 1 & 5 \\
\hline \multicolumn{14}{|l|}{ Presenting } \\
\hline$\% \mathrm{VA} \geq 3 / 60$ & \multicolumn{2}{|c|}{54} & \multicolumn{2}{|c|}{36} & \multicolumn{2}{|r|}{7} & & - & \multicolumn{2}{|c|}{1} & & 1 & 9 \\
\hline $\begin{array}{l}\text { \% Established } \\
\text { glaucoma in } \\
\text { other eye }\end{array}$ & \multicolumn{2}{|c|}{$37.7 \%$} & \multicolumn{2}{|c|}{$26.3 \%$} & \multicolumn{2}{|c|}{$33.3 \%$} & \multicolumn{2}{|c|}{$44.4 \%$} & \multicolumn{2}{|c|}{-} & & - & \multirow[t]{2}{*}{$66.7 \%$} \\
\hline $\begin{array}{l}\% \text { Severe } \\
\quad \text { hypertension }\end{array}$ & \multicolumn{2}{|c|}{$62.3 \%$} & & $.9 \%$ & & $.7 \%$ & & $3 \%$ & 100 & $0 \%$ & & - & \\
\hline
\end{tabular}

Table 2

Proportion of NVG subjects with presumed aetiological factors

\begin{tabular}{|c|c|c|c|c|c|c|c|c|}
\hline \multirow{3}{*}{$\begin{array}{l}\text { Presumed aetiological } \\
\quad \text { Factor }\end{array}$} & \multicolumn{8}{|c|}{ No of cases } \\
\hline & \multicolumn{2}{|c|}{ All } & \multicolumn{2}{|c|}{ HT } & \multicolumn{2}{|c|}{ DM } & \multicolumn{2}{|c|}{ Glaucoma } \\
\hline & No. & $(\%)$ & No. & $(\%)$ & No. & $(\%)$ & No. & $(\%)$ \\
\hline Retinal venous occlusion & 48 & 78.7 & 32 & 66.7 & 4 & 8.3 & 16 & 33.3 \\
\hline Central & 36 & 59.0 & 24 & 66.7 & 3 & 8.3 & 10 & 27.8 \\
\hline Hemispheric & 10 & 16.4 & 4 & 40 & & - & 4 & 40 \\
\hline Branch & 2 & 3.3 & 2 & 100 & & - & 2 & 100 \\
\hline Diabetic retinopathy & 1 & 1.6 & 1 & 100 & 1 & 100 & & - \\
\hline Retinal arterial occlusion & 1 & 1.6 & 1 & 100 & & - & & - \\
\hline \multicolumn{9}{|l|}{ Others } \\
\hline Couching & 7 & 11.5 & & & & & & \\
\hline Aphakia & 2 & 3.3 & & & & & & \\
\hline Chronic Uveitis & 1 & 1.6 & & & & & & \\
\hline Penetrating eye injury & 1 & 1.6 & & & & & & \\
\hline Total & 61 & & & & & & & \\
\hline
\end{tabular}


The predominant associated systemic factor in subjects who had retinal vascular occlusion were systemic hypertension in $38(62.3 \%)$ subjects, occurring predominantly in subjects with CRVO (24 out of 36), HCRVO (4 out of 10) BRVO (two out of two), CRAO in one subject.

Four subjects had diabetes mellitus. Of these, one had proliferative diabetic retinopathy; the other three subjects had central retinal vein occlusion and had systemic hypertension.

Ocular inflammation was identified as the aetiological factor associated with NVG. This was found in only one $(1.6 \%)$ subjects. The ocular inflammation was associated with a retina detachment in that subject. Seven (11.5\%) subjects had couching (a local method of dislocating the lens into the vitreous to treat blindness), two (3.3\%) had intracapsular cataract extraction but with vitreous loss. Only one subject had a penetrating eye injury.

Chronic glaucoma was newly diagnosed in the other eye in $23(37.7 \%)$ subjects with NVG. Of these 23 subjects who had chronic glaucoma in their other eye, $16(26.2 \%)$ have had retinal vein occlusion while seven $(11.5 \%)$ had couching done. Among these sixteen subjects the chronic glaucoma in their other eye was the open angles type in all cases.

There was visual impairment and severe visual impairment (visual acuity worse than $6 / 18$ and not less than $3 / 60)$ at presentation in $10(15.6 \%)$ eyes with NVG, while $54(84.4 \%)$ of affected eyes were blind (visual acuity $<3 / 60$ ) at presentation. Blindness at presentation occurred in 36 (92.3\%) eyes with CRVO, seven (70\%) eyes with HCRV, one eye with CRAO, one eye with diabetic retinopathy and in nine out of eleven eyes who had either couching, uveitis or injury.

\section{DISCUSSION}

Retinal vein occlusion was the leading ocular aetiological factor associated with NVG in this series of Nigerian African subjects. This finding agrees with several other clinical studies done in Caucasians (1-9). Chronic glaucoma was newly diagnosed in the other eye in more than a third of all subjects who had NVG.

Systemic arterial hypertension was the most frequent systemic disorder noted in subjects with NVG in this series. The diagnosis was made by clinical history and blood pressure measurement at the time of presentation in about two thirds of the subjects. The prevalence of hypertension in these subjects is much higher than the prevalence of hypertension in an age-matched general population, $(12,13)$ though the effect of selection bias might contribute to this.

Unlike other studies, (8) diabetic retinopathy (DR) was not a major associated factor with NVG in this population. DR was found in one subject and diabetes mellitus was a systemic disease found in only four subjects with NVG. The other three subjects, who had diabetes mellitus had no DR but had hypertension which was the presumed systemic cause of CRVO in their eyes. It is possible that diabetes mellitus was under diagnosed in these series as the diagnosis was based on urinalysis and clinical history alone. A glucose tolerance test might have made the number of subjects with diabetes mellitus higher.

Ocular inflammation was an infrequently associated condition leading to NVG unlike the findings by Hoskins (1). However, there might have been an under representation of ocular inflammation in subjects with NVG in this series. $11.5 \%$ subjects who presented with NVG had couching done. Couching was done to treat blindness in the population studied. The association between couching and NVG had not been previously reported. Most of the subjects who had couching also had glaucoma in their other eye. A high proportion of these subjects had systemic hypertension as well. Eyes that were couched could also have had inflammation. In eyes that were couched, it is therefore difficult to separate the relative importance of hypertension, glaucoma and inflammation in the pathogenesis of ocular neovascularisation. It could be that neovascularisation preceeded inflammation or vice versa. It is known that eyes with NVG can develop anterior chamber inflammation as a result of leaking vessels. Therefore in couched eyes, multiple factors such as inflammation, high intraocular pressure or even arterial vascular occlusion may interplay to cause NVG. Further studies are needed to evaluate the mechanism of ocular neovascularisation in a couched eye.

Retina arterial occlusion might have been under diagnosed in this series without concomitant arterial and venous obstruction can occur in an eye $(7,12)$. While venous obstruction could be diagnosed more easily by fundoscopy, arterial obstruction may 
require angiographic studies. The only subject whose diagnosis was CRAO in this series had severe hypertension.

Arteriosclerosis in hypertension have been suggested to be a major underlying factor causing vein occlusion by compression of veins either at the optic nerve head or on the retina (5). And in Africans, hypertensive retinopathy was found in $70 \%$ of 350 Nigerians with hypertension (14). In blacks, hypertension presents with higher pressures. Low socioeconomic status and poor access to medical care, poor compliance to therapy, all contribute to poor prognosis and increased complications among blacks $(12,13)$.

The relative low number of subjects with diabetes mellitus (DM) may reflect the relative higher prevalence of hypertension than that of DM in the general population (15), or may suggest a lower prevalence of ischaemic retinal diseases in the diabetic population in Nigerian Africans.

Among the 61 subjects in this study with an identifiable aetiological factor presumably causing NVG, 50(82\%) had associated posterior segment disease causing ischaemia. This was mainly retina venous obstruction $(78.7 \%)$. Those with no identifiable vaso-occlusive disease were two (3.3\%) with aphakia with vitreous wick syndrome, chronic uveitis one (1.6\%) and couching seven (11.5\%) and an eye with penetrating eye injury.

The subjects who presented with NVG in this series were more likely to have had ischaemic CRVO. NVG rarely results from non-ischaemic vascular occlusion $(3,4,11)$ Neovascular glaucoma is known to develop in $8 \%$ of all CRVO, and $67-82 \%$ of ischaemic CRVO $(3,4,10-12)$. The high prevalence of hypertension in these subjects suggest the condition to be a predisposing systemic factor associated with CRVO in this series.

However, the subjects studied might have had other diseases causing hyperviscosity. These were investigated. It is interesting however that no subject in this series had sickle cell disease even when the prevalence of sickle cell disease in the population is high (16). NVG has been reported to occur infrequently in subjects with proliferative sickle retinopathy (9). It has been suggested that the area of peripheral retinal ischaemia perhaps is smaller and hence less likely to elicit production of angiogenesis factor required to produce NVG $(9,17)$.
The age and sex distribution of subjects in these series are similar to those seen with vascular occlusive diseases $(6-7,16,17)$. The relative difference in sex ratio in subjects below 40 years and those above 40 years is noteworthy. There is a suggestion from this study that the associated factors with NVG in young subjects may be related to gender.

That $84.4 \%$ of eyes were blind on presentation underscores the severity of NVG. In developing countries, treatment of NVG are not readily available hence the loss of vision and sometimes the globe. There is therefore a need to tackle these known factors especially hypertension.

\section{CONCLUSION}

In this African population, medical conditions like hypertension and ocular conditions like glaucoma and couching were important associations found in subjects presenting with NVG. Both conditions were associated with retina vascular occlusive diseases.

Diabetes Mellitus was not frequently associated with the condition. The role of other conditions causing hyperviscosity in Africans need to be investigated.

NVG was a primary presentation of chronic glaucoma. Failure to diagnose this may lead to loss of vision in the other eye either from glaucomatous optic atrophy or a second NVG in the other eye.

Identification of these medical and ocular conditions and their treatment should reduce the prevalence of NVG and its consequences.

\section{REFERENCES}

1. Hoskins H.D. Jr. Neovascular glaucoma: Current concepts. Trans. Amer. Acad. Ophthalmol. Otolaryngol. 1974; 78: 330-333.

2. Garther S. and Henkind P. Neovascularisation of the iris (rubeosis iridis). Surv. Ophthalmol. 1978; 22: 291-312.

3. Knox D.L. 1schaemic ocular inflammation. Amer. J. Ophthalmol. 1985; 60: 995-1002.

4. Zegarra H., Gutman F.A. and Conforto J. The natural course of central retinal vein occlusion. Ophthalmol. 1979; 86: 1931-1942.

5. Hayreh S.S., Rojas P., Podhajsky P., Montaague P. and Woolson R.F. Ocular neovascularisation with retinal vascular occlusion-II. Incidence of ocular neovascularisation with retinal vein occlusion. Ophthalmol. 1983; 90: 488-506. 
6. Madaen P.H. Haemorrhagic glaucoma; comparative study in diabetic and non-diabetic patients. Brit. J. Ophthalmol. 1971; 55: 444-450.

7. Brown G.C., Magangal L.E., Simeone F.A., et al. Arterial obstruction and ocular neovascularisation. Ophthalmol. 1982; 89: 130-146.

8. Brown G.C., Magangal L.E., Schaschat A. and Shar H. Neovascular glaucoma aetiological considerations. Ophthalmol. 1984; 91: 31 5-319.

9. Dodson P.M., Kubicki A.J., Taylorr K.J. and Kritizingeer E.E. Medical conditions underlying recurrence of retinal vein occlusion. Brit. J. Ophthalmol. 1985; 69: 493-496.

10. Sinclair S.H. and Gragooudas E.S. Prognosis for rubeosis iridis following central retinal vein occlusion. Brit. J. Ophthalmol. 1979; 63: 735-743.

11. Magargal L.E., Donoso L.A. and Sanborn G.E. Retinal ischemia and risk of neovascularisation following central retinal vein obstruction. Ophthalmol. 1982; 89: 1241-1245.
12. Steyn K. et al. Hypertension in South African adults: Results from the demographic and health survey, 1998. J. Hypertension. 2001; 19: 1717-1725.

13. Lisk D.R. and Mc Eiven E.K. The significance and trend of hypertension related deaths in urban Sierian Leonean Africans. J. Human Hypertension. 1996; 10: 215-218.

14. Ladipo G.O. Hypertension retinopathy in Nigerians. A prospective clinical study of 350 cases. Trop. Geogr. Med. 1981; 33: 311-316.

15. Sobngwi E., Mauvais-Jarvis F., Vexiau P., Mbanyaa J.C. and Gautier J.F. Diabetes in Africans. Part 1: Epidemiology and clinical specific. Diabetes Metab. 2001; 27: 628-634.

16. Ibidapo M.O. and Akinyanju O.O. Acute sickle cell syndromes in Nigerian adults. Clin. Lab Haematol. 2000; 22: 15l-155.

17. Boniuk M. and Burton G.L. Unilateral glaucoma associated with sickle cell retinopathy. Trans. Amer. Acad. Ophthalmol. Otolaryngol. 1964; 38: 316-328. 